\title{
Seroprevalencia de anticuerpos contra Toxocara canis y factores de riesgo en niños, Ciudad Bolívar, Bogotá, D.C., 2000
}

\author{
Myriam Acero ${ }^{1}$, María Mercedes Muñoz ${ }^{2}$, Astrid Carolina Flórez ${ }^{3}$, Rubén Santiago Nicholls ${ }^{3}$ \\ ${ }^{1}$ Hospital Meissen, Bogotá, D.C., Colombia. \\ ${ }^{2}$ Clínica San Pedro Claver, Bogotá, D.C., Colombia. \\ ${ }^{2}$ Laboratorio de Parasitología, Instituto Nacional de Salud, Bogotá, D.C., Colombia.
}

La toxocariasis humana es una zoonosis causada por larvas de los nemátodos Toxocara canis y Toxocara cati, cuyo hábitat es el intestino de perros y gatos, respectivamente. Para estimar la prevalencia de anticuerpos contra $T$. canis y su relación con algunos factores de riesgo, se estudiaron 193 niños entre 4 y 14 años de edad procedentes de establecimientos oficiales de educación básica primaria de la localidad de Ciudad Bolívar en Bogotá durante el año 2000. Asimismo, para determinar la proporción de cachorros positivos al parásito T. canis, se estudiaron las muestras fecales de 21 perros que convivían con los niños en el momento del estudio. Se determinó la seropositividad de anticuerpos IgG por el método de ELISA y la prevalencia de infección por $T$. canis en heces de cachorros por el método modificado de McMaster. La prevalencia de seropositividad observada fue de 7,3\%, con una mayor proporción en niños menores de 5 años; sin embargo, el promedio de títulos aumentó con la edad. La prevalencia de infección en cachorros fue de $66,7 \%$. El factor de riesgo para la presencia de títulos de anticuerpos positivos contra $T$. canis que resultó estadísticamente significativo, fue el de no lavarse las manos antes de comer ( $R R=9,91$; IC $95 \%=1,27-77,21)$. Sin embargo, condiciones como la edad, el sexo, la geofagia y el incremento de la densidad poblacional canina pueden favorecer la transmisión de esta zoonosis en la localidad.

Palabras clave: toxocariasis, seropositividad, factores de riesgo, ELISA.

Toxocara canis: antibody seroprevalence and risk factors in children, Ciudad Bolívar, Bogotá, 2000

Human toxocariasis is a zoonotic infection caused by larvae of the nematode parasites Toxocara canis and Toxocara cati. The adult stages inhabit the intestine of dogs and cats, respectively. In order to estimate the prevalence of antibodies against $T$. canis and the relationship between seropositivity and some risk factors, a survey was conducted on 193 schoolchildren aged between 4 and 14 years from basic primary schools in the locality of Ciudad Bolivar, Bogotá. Fecal samples from 21 puppies were also examined to determine the prevalence of infected pet dogs. Serum IgG antibodies were determined by the indirect ELISA technique and parasitological examination of dog faeces was carried out by the modified McMaster method. The prevalence of antibodies was $7.3 \%$, with a higher prevalence in children under 5 years but with the mean titre increasing with age. The prevalence of infected puppies was $66.7 \%$. The only risk factor found to be correlated significantly with positive antibody titres was not washing hands before eating ( $R R=9.91$, IC $95 \%=1.27-77.21)$. However, several other factors such as age, gender, geophagy, and the increase in the density of the canines favored the transmission of this zoonosis.

Key words: toxocariasis, seropositivity, risk factor, ELISA.

Correspondencia:

A.C. Flórez, Grupo de Parasitología, INS, Avenida calle 26 No. 51-60, Bogotá, D.C., Colombia.

ins@hemagogus.ins.gov.co

Recibido: 26/04/01; aceptado: 6/08/01
Las zoonosis son entidades clínicas que han ido adquiriendo mayor importancia. En Latinoamérica, más de 380 millones de personas están expuestas directa o indirectamente a más de 150 enfermedades zoonóticas y 186 millones de latino- 
americanos han padecido una o más zoonosis durante el transcurso de sus vidas (1).

La toxocariasis humana es la más común de las zoonosis helmínticas, producida por estadios larvarios del nemátodo Toxocara canis de los cánidos y, en menor grado, Toxocara cati de los gatos. Esta infección en el hombre se considera aberrante, ya que no es su hospedero natural sino un hospedero paraténico en el que se desencadena una migración de los estadios larvarios de este parásito sin completar su ciclo de vida ni alcanzar su estado adulto, produciendo una infección larvaria sistémica que puede presentarse como larva migrans visceral (LMV), larva migrans ocular (LMO) o toxocariasis encubierta $(2,3)$.

En los seres humanos, la toxocariasis se adquiere por ingestión accidental de huevos embrionados de materias fecales provenientes de cánidos infectados, dispersas en suelos de jardines, parques y lugares públicos $(1,3,4)$.

Estudios serológicos indican que la infección, mas no la enfermedad, se encuentra ampliamente distribuida en el mundo. En 1979, más de 1.900 casos de toxocariasis humana habían sido reportados en 50 países del mundo (5). En 1983, se reportó una seroprevalencia de $2,8 \%$ en un estudio con 8.547 sueros de sujetos representativos de toda la población de Estados Unidos, con una mayor prevalencia obtenida en niños menores de 12 años, en personas de raza negra de todas las edades y entre personas de nivel socioeconómico bajo $(6,7)$. En algunos países industrializados de Europa, se han revelado seroprevalencias de 2,5 a $5 \%$ en adultos urbanos, pero en población rural se han reportado tasas mayores a $17 \%$ en una población de agricultores suecos, $37 \%$ en campesinos de Francia y más del $40 \%$ en niños irlandeses (8).

La frecuencia de la enfermedad en Colombia se conoce desde 1966 con un primer caso de toxocariasis visceral o larva migrans visceral en una niña de 14 años proveniente de las orillas del río Cauca $(9,10)$. En 1981, la Fundación Oftalmológica Nacional y el Instituto Nacional de Salud determinaron títulos de anticuerpos contra
T. canis en 185 estudiantes de dos escuelas de Bogotá; se encontró una prevalencia de $13,5 \%$; además, en 200 muestras de materia fecal de cánidos provenientes de parques de la ciudad se encontraron $28(14 \%)$ con huevos de T. canis (11). En otro estudio realizado en Bogotá por el Instituto Nacional de Salud en una comunidad de bajo nivel económico, de 207 personas se encontraron títulos positivos en $47,5 \%$ y de 39 cachorros, $46,3 \%$ fueron positivos (12).

La Organización Panamericana de la Salud (OPS) estima para las grandes ciudades latinoamericanas una relación de 10 caninos por cada 100 personas y, para los estratos más bajos, de 15 caninos por cada 100 personas. Sin embargo, en un estudio realizado por el área de investigaciones de la Secretaría Distrital de Salud durante 1997 y 1998 sobre la determinación de niveles de anticuerpos contra el virus de la rabia en caninos de la localidad de Ciudad Bolívar en Bogotá, de un total de 148 propietarios de caninos se determinó una relación de 25 caninos por cada 100 habitantes. Este hecho, que supera lo estimado por la OPS, sumado al censo de 1997 de la localidad de Ciudad Bolívar realizado por el DANE, que arrojó una población total de 498.177 habitantes, siendo la localidad de Bogotá con mayor proporción de necesidades básicas insatisfechas (NBI), 39,11\%, una población canina con una tasa de crecimiento tan alta, rápida y desordenada, un inadecuado mantenimiento canino y una estrecha relación hombre-perro, hace que sea de gran relevancia determinar la seroprevalencia de anticuerpos contra T. canis en niños de establecimientos educativos de esta localidad.

En el presente estudio, se determinó la seroprevalencia de anticuerpos contra T. canis en niños de la localidad de Ciudad Bolivar y se analizó su relación con algunos factores de riesgo como la edad, el sexo, los hábitos y las actitudes, la convivencia con perros teniendo en cuenta su número por vivienda, su edad y la positividad a $T$. canis, con el fin de contribuir a proponer programas de prevención y control de enfermedades zoonóticas a través del conocimiento del comportamiento epidemiológico de la toxocariasis. 


\section{Materiales y métodos}

Población, variables del estudio y recolección de datos: se realizó un estudio observacional descriptivo transversal para estimar la seroprevalencia de anticuerpos contra T. canis en una población de estudio constituida por 193 niños de 6 establecimientos educativos oficiales escogidos por selección aleatoria durante el año 2000 , que representaban a la población de 31.557 niños de educación básica primaria de la localidad de Ciudad Bolívar. Se exploró la relación con algunos factores de riesgo como sexo, edad, consumo de tierra, pasto y uñas, lavado de manos, presencia de perros en la vivienda, presencia de cachorros en la vivienda desde el nacimiento del niño y su procedencia.

A continuación se aplicó al padre, la madre o el acudiente del niño una encuesta para recoger información sobre los datos personales del niño, los hábitos, las actitudes y la convivencia con perros en el momento del estudio. Además, se solicitó el debido consentimiento para la extracción de sangre venosa, procedimiento considerado como de riesgo mínimo, teniendo en cuenta las normas sobre aspectos éticos de investigación en humanos, según la Resolución 008430 del Ministerio de Salud.

Muestras: se realizó la toma de $5 \mathrm{ml}$ de sangre venosa, aproximadamente, por niño en tubos sin anticoagulante, debidamente rotulados. Los sueros obtenidos por centrifugación se almacenaron a $-20^{\circ} \mathrm{C}$ hasta el momento de su procesamiento.

Antígeno: el antígeno excretor-secretor (ES) se obtuvo mediante cultivo in vitro de larvas de T. canis en estadio larvario II, obtenidas por eclosión de huevos embrionados (13) y se almacenó a $-70^{\circ} \mathrm{C}$ hasta el momento de su utilización.

Procesamiento de muestras: se utilizó para su procesamiento la técnica de ELISA, en la que inicialmente, por 18 horas a $4{ }^{\circ} \mathrm{C}$, se realizó la sensibilización de las placas de poliestireno mediante adición del antígeno ES, seguida de un lavado por triplicado con solución amortiguadora PBS-Tween (PBS-T), pH 7,4 para eliminar la solución antigénica. Posteriormente, se adicionaron las muestras de sueros por analizar en dilución 1:800 durante 2 horas, tiempo al cabo del cual se procedió al lavado de la placa en la forma ya descrita. Seguidamente, se adicionó como conjugado enzimático anti-IgG humana unida a fosfatasa alcalina, a una dilución 1:12.000 durante 18 horas a $4^{\circ} \mathrm{C}$. Transcurrido este tiempo, las placas se lavaron con solución amortiguadora PBS-T y se adicionó el sustrato cromogénico paranitrofenilfosfato-dietanolamina durante 30 minutos a temperatura ambiente. La reacción enzimática fue, entonces, detenida mediante adición de $25 \mu$ le $\mathrm{NaOH} 3 \mathrm{M}(14,15)$.

Lectura e interpretación: la lectura se realizó en un fotocolorímetro a una longitud de onda de 405 $\mathrm{nm}$. Se obtuvo un promedio de tres lecturas en absorbancia o densidad óptica (DO) para cada muestra. Se consideró muestra positiva para anticuerpos contra $T$. canis aquélla con densidad óptica mayor al punto de corte $(0,390)$ y como muestra negativa, aquélla con absorbancia menor al mismo punto (15).

Procesamiento de muestras de cachorros: los padres que afirmaron poseer perros cachorros en la vivienda del niño, procedieron a recoger las respectivas muestras de materia fecal, que fueron procesadas por la técnica modificada de McMaster para determinar la proporción de huevos de caninos infectados con $T$. canis y su carga parasitaria (16). Para el procesamiento de esta técnica, se obtuvo una mezcla homogénea de $2 \mathrm{~g}$ de materia fecal de cachorro y $28 \mathrm{ml}$ de agua destilada, la cual fue sometida a filtración y centrifugación para obtener un sobrenadante al que se le adicionó la solución de McMaster. Seguidamente, se tomó cantidad suficiente para llenar la cámara y proceder a realizar la búsqueda de huevos bajo microscopía de luz.

Análisis estadístico: para variables cuantitativas, se utilizó la media aritmética, la mediana y la desviación estándar. La prevalencia de anticuerpos contra $T$. canis se estimó mediante proporciones de prevalencia general. La proporción de T. canis en materia fecal de cachorros se determinó mediante proporción general y se agrupó según el nivel de carga parasitaria. Para establecer si existían diferencias en la prevalencia de anticuerpos según los diferentes factores de riesgo incluidos en el estudio, se realizaron pruebas estadísticas 
de diferencias de proporciones y para detectar la influencia del azar se utilizaron pruebas de significación con base en la distribución de ji cuadrado. En los casos en que la frecuencia esperada era menor de 5 , se realizó la prueba exacta de Fisher. Para este análisis, se empleó el programa Epilnfo versión 6,04 (17).

\section{Resultados}

La edad promedio de los 193 niños participantes en el estudio serológico fue de 8,4 años; 114 $(59,1 \%)$ eran niños y $79(40,9 \%)$ niñas. Del total de muestras, $47(24,4 \%)$ fueron tomadas en establecimientos educativos del área rural y 146 $(75,6 \%)$ en establecimientos educativos de la zona urbana.

Se obtuvo, por medio de la prueba de ELISA, una seroprevalencia general de $7,3 \%(n=14)$. La mayoría de muestras positivas para anticuerpos contra T. canis correspondió al rango de DO de 0,390 a $0,499(n=8)$ y se presentaron densidades ópticas mayores de 0,650 en $2,1 \%$ de los niños $(n=4)$.

La mayor prevalencia de anticuerpos contra $T$. canis se obtuvo en las muestras de niños menores de 5 años; en cuanto al sexo, los niños presentaron una mayor prevalencia $(7,9 \%)$ comparada con la obtenida en las niñas (6,3\%), aunque la diferencia no fue estadísticamente significativa (figura 1).

Al observar los resultados del promedio de densidades ópticas totales correspondientes a títulos de anticuerpos (positivos y negativos) del estudio, se observa que estos variaron entre 0,002 y 0,874 , con un incremento gradual con la edad

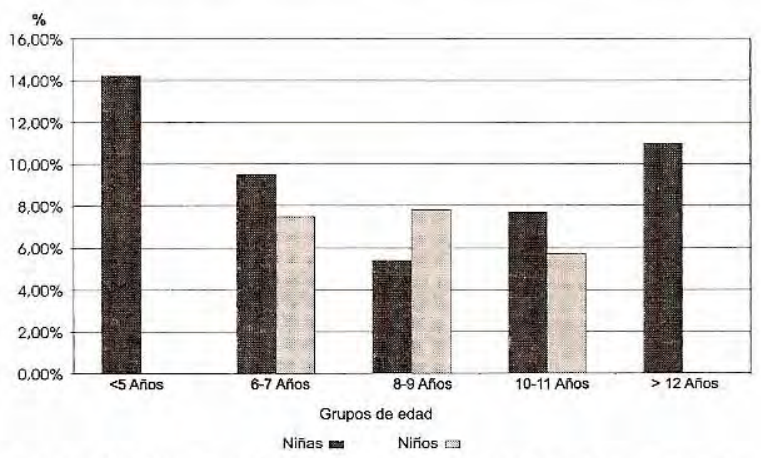

Figura 1. Prevalencia de anticuerpos contra $T$. canis según edad y sexo. en casi todos los grupos, excepto en el grupo de 6 a 7 años donde se observó un ligero descenso.

Los valores de densidad óptica de la población seropositiva variaron entre 0,390 a 0,874 con un promedio de 0,547 ; el promedio más alto de densidades ópticas se observó en el rango de edad de 8 a 9 años (figura 2); según el sexo, en los niños fue más alto $(0,633)$ que en las niñas $(0,540)$ y con respecto al establecimiento educativo el promedio de densidades ópticas fue superior en la zona rural $(0,683)$ en relación con la zona urbana $(0,550)$.

\section{Hábitos de la población}

Teniendo en cuenta la encuesta aplicada a los padres $o$ acudientes de los niños pertenecientes al estudio, se realizó un análisis de las características y factores de riesgo en 176 encuestas que contenían todas las respuestas correspondientes a sexo, edad, geofagia o consumo de tierra, consumo de pasto, onicofagia o hábito de comer uñas, lavado de manos, presencia de perros y cachorros en la vivienda y procedencia (cuadro 1). Según el análisis del cuadro 1, se pudo determinar con un intervalo de confianza del $95 \%$ que no existen diferencias estadísticamente significativas en este estudio, entre la prevalencia de anticuerpos contra T. canis y las diferentes variables del estudio, con excepción del hábito de no lavarse las manos antes de comer que resultó significativamente asociado con la presencia de anticuerpos contra $T$. canis ( $\mathrm{RP}=9,91 ; \chi^{2}=12.84, \mathrm{p}=0,0016, \mathrm{gl}=2$ ). La edad $\mathrm{y}$ la ingestión de tierra y pasto no constituyeron factores de riesgo para la infección (cuadro 1). Se

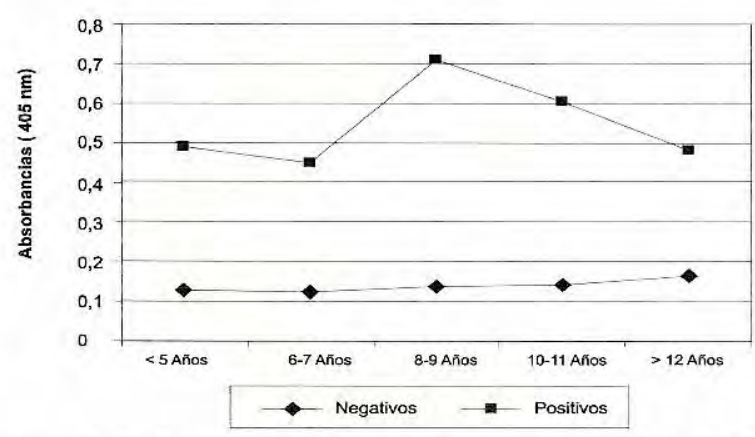

Figura 2. Promedio de valores de densidad óptica de anticuerpos contra T. canis por grupos de edad. 
Cuadro 1. Características y factores de riesgo en niños, según reactividad serológica a $T$. canis.

\begin{tabular}{|c|c|c|c|c|c|c|c|c|}
\hline \multirow[b]{2}{*}{ Factores de riesgo } & \multicolumn{2}{|c|}{$\begin{array}{c}\text { Positivos } \\
n=13\end{array}$} & \multicolumn{2}{|c|}{$\begin{array}{c}\text { Negativos } \\
n=163\end{array}$} & \multirow[b]{2}{*}{ Total } & \multirow[b]{2}{*}{$\mathbf{R P}^{*}$} & \multirow[b]{2}{*}{ IC $95 \%$} & \multirow[b]{2}{*}{$p$} \\
\hline & $\bar{n}$ & $\%$ & $n$ & $\%$ & & & & \\
\hline \multicolumn{9}{|l|}{ Sexo } \\
\hline Femenino & 5 & 6,8 & 68 & 93,2 & 73 & 0,88 & $0,30-2,59$ & 0,81 \\
\hline Masculino & 8 & 7,8 & 95 & 92,2 & 103 & & & \\
\hline \multicolumn{9}{|l|}{ Edad } \\
\hline 2-5 años & 1 & 11,1 & 8 & 88,9 & 9 & 1,55 & $0,23-10,62$ & 0,66 \\
\hline$>5$ años & 1 & 7,2 & 155 & 92,8 & 167 & & & \\
\hline \multicolumn{9}{|l|}{ Consumo de tierra } \\
\hline Sí & 7 & 13,0 & 47 & 87,0 & 54 & 2,64 & $0,93-7,47$ & 0,05 \\
\hline No & 6 & 4,9 & 116 & 95,1 & 122 & & & \\
\hline \multicolumn{9}{|l|}{ Consumo de pasto } \\
\hline Sí & 3 & 13,6 & 19 & 86,4 & 22 & 2,10 & $0,63-7,05$ & 0,23 \\
\hline No & 10 & 6,5 & 144 & 93,5 & 154 & & & \\
\hline \multicolumn{9}{|l|}{ Consumo de uñas } \\
\hline Sí & 6 & 7,0 & 80 & 93,0 & 86 & 0,90 & $0,31-2,56$ & 0,83 \\
\hline No & 7 & 7,8 & 83 & 92,2 & 90 & & & \\
\hline \multicolumn{9}{|l|}{ Lavado de manos } \\
\hline Siempre & 1 & 2,6 & 379 & 7,4 & 38 & 1 & & \\
\hline Algunas veces & 6 & 5,2 & 109 & 94,8 & 115 & 1,98 & $0,25-15,95$ & 0,5 \\
\hline Nunca & 6 & 26,1 & 17 & 73,9 & 23 & 9,91 & $1,27-77,21$ & $<0,05$ \\
\hline \multicolumn{9}{|l|}{$\begin{array}{l}\text { Presencia de perros } \\
\text { en la vivienda }\end{array}$} \\
\hline Sí & 8 & 7,4 & 100 & 92,6 & 108 & 1,01 & $0,34-2,95$ & 0,98 \\
\hline No & 5 & 7,3 & 63 & 92,7 & 68 & & & \\
\hline \multicolumn{9}{|l|}{$\begin{array}{l}\text { Presencia de cachorros } \\
\text { en la vivienda }\end{array}$} \\
\hline Sí & 3 & 11,5 & 23 & 88,5 & 26 & 1,73 & $0,51-5,87$ & 0,38 \\
\hline No & 10 & 6,7 & 140 & 93,3 & 150 & & & \\
\hline \multicolumn{9}{|l|}{$\begin{array}{l}\text { Presencia de cachorros } \\
\text { desde que nació el niño }\end{array}$} \\
\hline Sí & 9 & 7,8 & 107 & 92,2 & 116 & 1,16 & $0,37-3,62$ & 0,79 \\
\hline No & 4 & 6,7 & 56 & 93,3 & 60 & & & \\
\hline \multicolumn{9}{|l|}{ Procedencia del niño } \\
\hline Zona urbana & 11 & 8,2 & 124 & 91,8 & 135 & 1,67 & $0,3-7,23$ & 0,48 \\
\hline Zona rural & 2 & 4,9 & 39 & 95,1 & 41 & & & \\
\hline
\end{tabular}

*RP: razón de prevalencia (riesgo de un individuo de ser positivo o no a la prueba de T. canis)

halló un promedio de perros por vivienda de 1,1 con un rango que varió entre 1 a 5 perros. Ni la presencia de perros en la vivienda ni la convivencia con cachorros desde el momento de su nacimiento fueron significativos como factores de riesgo; sin embargo, se presentó un mayor riesgo en aquellas viviendas donde existían perros $(\mathrm{RP}=1,01)$ y un riesgo mayor en aquéllas donde existían cachorros desde el nacimiento de los niños $(\mathrm{RP}=1,16)$ (cuadro 1). Tampoco fue estadísticamente significativo el riesgo de adquirir la infección de acuerdo con la procedencia, urbana o rural, de los niños (cuadro 1).

\section{Infección por $\boldsymbol{T}$. canis en los caninos}

De 40 cachorros que convivían con los niños en el momento del estudio, se recogieron 21 muestras de materia fecal, teniendo en cuenta que en los casos en donde existía más de un cachorro por vivienda, se seleccionó aleatoriamente uno de ellos y 10 niños no entregaron las muestras de sus cachorros o éstas fueron recogidas en malas condiciones para su procesamiento. Se observaron huevos de T. canis en 14 muestras $(66,7 \%$ y es necesario resaltar que 7 de estas muestras $(33,3 \%)$ presentaron 
Cuadro 2. Nivel de carga parasitaria de muestras caninas.

\begin{tabular}{ccc}
\hline $\begin{array}{c}\text { No. de muestras } \\
\text { positivas }\end{array}$ & $\begin{array}{c}\text { Proporción } \\
\mathbf{( \% )}\end{array}$ & $\begin{array}{c}\text { Carga parasitaria } \\
\mathbf{( h / g})^{\star}\end{array}$ \\
\hline 11 & 78,6 & Leve (1 a 1.999) \\
2 & 14,3 & Moderada \\
& & $(2.000$ a 9.999$)$ \\
1 & 7,1 & Grave $(>10.000)$ \\
\hline
\end{tabular}

*h/g: huevos por gramo de materia fecal

huevos embrionados potencialmente infectantes. El nivel de carga parasitaria de las muestras se presenta en el cuadro 2.

\section{Discusión}

En la mayoría de los países, incluso Colombia, los animales domésticos como el perro y el gato son los preferidos como mascotas, a pesar de lo cual existe en la población un desconocimiento del importante papel que estos animales tienen en el ciclo evolutivo y en la transmisión de la toxocariasis y otras zoonosis. Entre los factores de riesgo que favorecen la toxocariasis, se encuentran la alta población canina y las deficientes condiciones higiénico-sanitarias (4).

La seroprevalencia de anticuerpos contra $T$. canis obtenida en el estudio, $7,3 \%$, fue menor en comparación con los valores obtenidos en los estudios de niños con edades similares realizados en otros países, pero mayor que la seroprevalencia de un estudio elaborado en Japón en 1983 con 83 niños en el cual se determinó una tasa de $3,6 \%$ y similar a la de un estudio realizado en 1989 en Salamanca (España) con 90 niños en el cual la prevalencia fue del $7,0 \%(18)$. Sin embargo, para la comparación de resultados con otros estudios se deben tener en cuenta varios aspectos de tipo metodológico como los rangos de edad seleccionados, el punto de corte (DO) de títulos positivos y las diferencias en la metodología de la prueba de ELISA incluyendo la preparación del antígeno.

Con relación a otros estudios realizados en Colombia y en especial en Bogotá, resulta difícil hacer comparaciones ya que, de los dos estudios informados, uno de ellos encontró seroprevalencias de $47,5 \%$ en población de todas las edades con títulos significativamente más altos en el grupo de edad de 0 a 4 años que en el de 15 a 44 años (12), similar a lo encontrado en el presente estudio. El otro estudio fue realizado en población escolar de educación secundaria con una edad promedio de 13,6 años y se encontró un $13,5 \%$ de muestras positivas (11).

La seroprevalencia disminuyó a medida que la edad aumentaba, contrario a lo que se observó para los valores de densidad óptica en general, los cuales tendieron a incrementarse con la edad. En un estudio realizado en 1995 en Dublin (Irlanda), en una muestra de 2.129 sueros en población de 4 a 18 años, se encontró un resultado similar en el cual el promedio de títulos de anticuerpos aumentaba con la edad, lo cual sugiere que a mayor edad se presentaba una progresiva elevación de los títulos medios, como resultado de persistencia en la infección, pues la larva puede permanecer hasta 10 años en los tejidos estimulando el sistema inmune (18).

La seroprevalencia más alta encontrada en los niños menores de 5 años puede estar relacionada con circunstancias como el hecho de que los niños más pequeños están con frecuencia en mayor contacto con el suelo, tienen el hábito de llevarse los dedos a la boca e ingieren diferentes elementos que encuentran en el medio. Es así como en esta investigación, a pesar de que no se encontró una asociación estadísticamente significativa entre la ingestión de tierra (geofagia) y la infección por T. canis $(\mathrm{RP}=2,64$; IC $95 \%=0,93-7,47)$, la prevalencia de infección fue superior en los niños que sí tenían este hábito que en los que no, siendo el grupo de edad en donde se presentó con mayor frecuencia el de los niños menores de 5 años. En otro estudio en el cual se encontró una seropositividad de anticuerpos contra T. canis de $10,5 \%$, se demostró una asociación estadística-mente significativa con la geofagia como factor de riesgo de la infección (18). Cabe considerar la posibilidad de que con una mayor población de encuestados el valor de $p$ hubiese sido estadísticamente significativo. También es factible que el hábito sea más frecuente pero que los niños lo nieguen, no lo recuerden o que sus padres no lo hayan observado.

El hecho de que los niños presenten mayor seroprevalencia que las niñas ha sido reportado 
previamente (18); sin embargo, en este estudio no se encontraron diferencias significativas, pero la seropositividad y el promedio de densidad óptica de títulos positivos fueron mayores en los niños en relación con las niñas, posiblemente por el hecho de que a las niñas se les presta mayor atención respecto al tiempo de permanencia en la calle y por los juegos propios de su género (19). Aunque la diferencia no fue estadísticamente significativa, el hábito de geofagia fue un mayor factor de riesgo para la infección en niños que en niñas.

Aunque la ubicación rural o urbana del establecimiento educativo no fue un factor de riesgo significativo para seropositividad, la mayor seroprevalencia se obtuvo en una escuela del área urbana. Esto podría tener relación con el hecho que en ésta se encontraba la población de menor edad, en la cual se encontró la mayor seroprevalencia.

Sin embargo, el mayor promedio de densidad óptica de títulos positivos, el porcentaje más alto de geofagia, la mayor convivencia con cachorros alguna vez en la vida y la tenencia de perros en el momento del estudio se obtuvieron en los niños de la escuela rural. Lo anterior hace suponer que, especialmente por comportamientos como la geofagia en los niños, la alta densidad de perros y cachorros y la forma de mantenimiento de los mismos sin limitaciones de espacio ni sitios específicos para defecar y sin ningún control en la recolección de excrementos, las zonas rurales se convierten en espacios que benefician la transmisión de esta zoonosis, pero en la zona urbana el riesgo de la infección puede ser mayor si se tiene en cuenta que son los parques y los sitios públicos los preferidos para las deposiciones de las mascotas y, además, los niños pueden entrar en contacto más fácilmente con las excretas de los perros dado que éstas permanecen por varios días en las terrazas o patios de las casas que, a la vez, son los sitios de juego de los niños.

En cuanto al no lavarse las manos antes de comer y su relación con la seropositividad, se encontró asociación significativa siendo mayor la proporción en niños que con menor frecuencia o nunca se lavan las manos. En los niños más pequeños, este hábito está supeditado a la vigilancia y orden por parte de los padres para hacerlo y no es fácil que se convierta en un hábito. Los niños pueden tener contacto con materia fecal en los parques, en los jardines y en las calles al jugar con arena o tierra lo que, junto con la carencia del hábito de lavarse las manos, favorece la transmisión de la infección. En Chile, en un estudio realizado a partir de casos sintomáticos de toxocariasis, se encontraron diferencias estadísticamente significativas entre los casos y los controles según esta variable (20). En 1998, en un estudio de seroprevalencia de anticuerpos en médicos veterinarios dedicados a la medicina canina y felina, se demostró asociación estadística entre el no uso de guantes durante la consulta y la seroprevalencia (21).

La elevada proporción $(66,7 \%)$ de cachorros positivos a huevos de $T$. canis observada, es de gran relevancia si, además, se tiene en cuenta que $33,3 \%$ de estas muestras presentaban huevos embrionados, generando un alto riesgo si el manejo de las excretas de los animales en las viviendas no es el más adecuado.

El análisis de la razón de prevalencia entre viviendas y la tenencia o no de cachorros menores de 1 año, mostró que existe mayor riesgo $(\mathrm{RP}=1,73$ con $\mathrm{IC} 95 \%=0,51-5,87)$ de ser positivo a $T$. canis en las viviendas que sí poseían cachorros, aunque la diferencia no fue estadísticamente significativa. Diferentes estudios han encontrado proporciones altas del parásito en los cachorros. En 1990, se reportó $43 \%$ de positividad en muestras de materia fecal de cachorros de un barrio de nivel económico bajo de Bogotá (11). En un estudio realizado por el CDC de Atlanta en 1994 en perros de 7 semanas a 3 meses de edad, la infección por Toxocara fue cercana al $100 \%$ y aunque el $80 \%$ de los cachorros menores de 6 semanas tenían vermes en el intestino, sólo el $20 \%$ fue positivo a T. canis en el examen fecal, concluyendo que en cachorros muy jóvenes son frecuentes los falsos negativos $(22,23)$.

En general, sería importante avanzar con el conocimiento de esta zoonosis a través de estudios sobre el grado de contaminación por $T$. canis en parques y áreas de recreación infantil, ya que, a pesar de la prohibición a los propietarios de perros de permitir que éstos defequen en los 
parques y la obligación de recoger estos excrementos, en la práctica esta medida pocas veces se cumple. Teniendo en cuenta la importancia de esta zoonosis como problema de salud pública en la población infantil, con este mayor conocimiento se podrían adoptar medidas de prevención tendientes a controlar su transmisión.

\section{Agradecimientos}

Los autores expresan sus agradecimientos a la comunidad de la localidad de Ciudad Bolívar de Bogotá por su gran contribución en el desarrollo de este estudio y a Mauricio Beltrán, jefe de la División Laboratorio Nacional de Referencia del INS.

\section{Referencias}

1. Acha P, Szyfres B. Zoonosis y enfermedades transmisibles comunes al hombre y a los animales. Segunda edición. Washington, D.C.: Organización Panamericana de la Salud; 1988. p.844.

2. Laufer M, Tolan R. Toxocariasis, julio 2000. 10 p. Disponible en: http://www.emedicine.com/PED/topic 2270.htm.

3. Schenone H. Parasitosis humanas que pueden ser causadas o transmitidas por mascotas domésticas en Chile. Bol Chil Parasitol 1987;42:16-23.

4. Botero D, Restrepo M. Parasitosis humanas. Segunda edición. Medellín: Corporación para Investigaciones Biológicas; 1992. p.309-12.

5. Herrmann N, Glickman L, Schantz P, Weston M, Domanski L. Seroprevalence of zoonotic toxocariasis in the United States: 1971-1973. Amer J Epidemiol 1985; 122:890-6.

6. Glickman L, Schantz P. Epidemiology and pathogenesis of zoonotic toxocariasis. Epidemiol Rev 1981;3:23050.

7. Glickman L, Magnaval J, Domanski L, Shofer F, Lauria S, Gottstein B, Brochier B. Visceral larva migrans in French adults: a new disease syndrome? Amer J Epidemiol 1987;125:1019-34.

8. Magnaval J, Galindo V. Human toxocara infection of the central nervous system and neurological disorders: a case-control study. Parasitology 1997;115(Supl. 5): 537-43.

9. Correa P, González M, D’Alessandro A. Primer caso colombiano de toxocariosis. Antioquia Méd 1966;16: 489-97.
10. Escobar Melguizo JA, Little MD. Caso clínico con confirmación parasitológica de larva migratoria visceral por Toxocara canis en Colombia. Antioquia Méd 1966; 16:499-507.

11. Rodríguez A, Silva JC, Corredor A. Toxocariasis en la población asintomática. Cirugía 1986;1:79-80.

12. Agudelo C, Villarreal E, Cáceres E, López C, Elhach J, Ramírez N, Hernández CA, Corredor A. Human and dogs Toxocara canis infection in a poor neighbourhood in Bogotá. Mem Inst Oswaldo Cruz 1990; 85:75-8.

13. Savigny DH. In vitro maintenance of Toxocara canis larvae and a simple method for the production of Toxocara ES antigen for use in serodiagnostic tests for visceral larva migrans. J Parasitol 1975;61:781-2.

14. Voller A, Bidwell DE, Barlett A. Enzyme inmunoassay diagnostic medicine; theory and practice. Bull WHO 1976;53:55-6.

15. Villarreal E, López C, Corredor A. Preparación de antígeno secretorio de Toxocara canis para el diagnóstico serológico en humanos por el método de ELISA. Biomédica 1987;17(Supl.1):71-2.

16. Soulsby EJ. Parasitología y enfermedades parasitarias en los animales domésticos. México, D.F.: Nueva Editorial Interamericana; 1987. p.820-3.

17. CDC, Centers For Disease Control and Prevention. Epi Info 6, Versión 6.04. Atlanta GA: Centers For Disease Control and Prevention; 1996.

18. Holland CV. Epidemiology of toxocariasis in schoolchildren. Parasitology 1995;110:535-45.

19. Conde L, Muro A, Simon F. Epidemiological studies on toxocariasis and visceral larva migrans in a zone of Western Spain. Ann Trop Med Parasitol 1989;83: $615-20$

20. Noemi I, Rugiero E, Viovy A, Cortes P, Cerva J, Gonzales M, Back S, Gottlieb B, Herrera M, Cordovez J. Seroepidemiología familiar de la toxocariasis. Bol Chil Parasitol 1994;49:52-9.

21. Avellaneda D, Vinck G. Estudio descriptivo de la infección por Toxocara canis en médicos veterinarios dedicados a la medicina canina y felina en Santa $\mathrm{Fe}$ de Bogotá en 1998 (tesis). Bogotá, D.C.: Universidad de la Salle; 1998.

22. Schantz P. Toxocara larva migrans now. Am J Trop Med Hyg 1989;41(Supl.II):21-34.

23. Schantz P, Glickman L. Ascáridos de perros y gatos: un problema de salud pública y de medicina veterinaria. Bol Oficina Sanit Panam 1983;571-85. 